Portland State University

PDXScholar

6-10-2004

\title{
Approximate Thermodynamic State Relations in Partially lonized Gas Mixtures
}

John D. Ramshaw

Portland State University, jdramshaw@yahoo.com

Follow this and additional works at: https://pdxscholar.library.pdx.edu/phy_fac

Part of the Physics Commons

Let us know how access to this document benefits you.

Citation Details

J. D. Ramshaw, "Approximate thermodynamic state relations in partially ionized gas mixtures," Phys. Plasmas 11, 3572 (2004).

This Article is brought to you for free and open access. It has been accepted for inclusion in Physics Faculty Publications and Presentations by an authorized administrator of PDXScholar. Please contact us if we can make this document more accessible: pdxscholar@pdx.edu. 


\title{
Approximate thermodynamic state relations in partially ionized gas mixtures
}

\author{
John D. Ramshaw \\ Lawrence Livermore National Laboratory, University of California, P. O. Box 808, L-095, Livermore, \\ California 94551
}

(Received 11 February 2004; accepted 5 April 2004; published online 10 June 2004)

\begin{abstract}
Thermodynamic state relations for mixtures of partially ionized nonideal gases are often approximated by artificially partitioning the mixture into compartments or subvolumes occupied by the pure partially ionized constituent gases, and requiring these subvolumes to be in temperature and pressure equilibrium. This intuitively reasonable procedure is easily shown to reproduce the correct thermal and caloric state equations for a mixture of neutral (nonionized) ideal gases. The purpose of this paper is to point out that (a) this procedure leads to incorrect state equations for a mixture of partially ionized ideal gases, whereas (b) the alternative procedure of requiring that the subvolumes all have the same temperature and free electron density reproduces the correct thermal and caloric state equations for such a mixture. These results readily generalize to the case of partially degenerate and/or relativistic electrons, to a common approximation used to represent pressure ionization effects, and to two-temperature plasmas. This suggests that equating the subvolume electron number densities or chemical potentials instead of pressures is likely to provide a more accurate approximation in nonideal plasma mixtures. (C) 2004 American Institute of Physics.
\end{abstract}

[DOI: $10.1063 / 1.1758717]$

\section{INTRODUCTION}

Multicomponent hydrodynamics calculations require thermodynamic state relations for material mixtures. Unfortunately, it is rarely feasible to construct accurate state relations for multicomponent atomic mixtures of interacting materials, and even less feasible to employ them in practice. In lieu of this, it is necessary to approximate the state relations of the mixture in terms of those of the pure materials of which it is composed. The question then arises of how to construct the best or most accurate approximations of this type.

The only obvious way to proceed is to regard the mixture as being artificially partitioned or separated into its constituent components or materials, with material $k$ regarded as being confined by itself within a compartment or subvolume with volume fraction $\alpha_{k}$ and possessing a fraction $\beta_{k}$ of the total internal energy of the mixture, where of course $\Sigma_{k} \alpha_{k}$ $=\Sigma_{k} \beta_{k}=1$. Thus, in a mixture of $N$ materials $N-1$ of the variables $\alpha_{k}$ may be independently varied, and another $N$ -1 of the variables $\beta_{k}$, for a total of $2 N-2$ such variables. In most hydrodynamical situations, the natural independent thermodynamic variables for the mixture are the partial mass densities $\rho_{k}$ and the specific internal energy (energy per unit mass) $I$, which is not purely thermal but also includes chemical/ionization energy. The values of $\rho_{k}$ and $I$ are therefore presumed known. The mass density of pure material $k$ within its subvolume is then given by

$$
\widetilde{\rho}_{k}=\rho_{k} / \alpha_{k} .
$$

The internal energy density (energy per unit volume) of the mixture is $\rho I$, where $\rho=\Sigma_{k} \rho_{k}$ is the total mass density. According to the definition of $\beta_{k}$, the internal energy of mate- rial $k$ per unit total volume is then simply $\beta_{k} \rho I$, so the internal energy density of material $k$ within its subvolume is $\beta_{k} \rho I / \alpha_{k}=\widetilde{\rho}_{k} I_{k}$, where $I_{k}$ is the specific internal energy of material $k$. It follows that

$$
\rho_{k} I_{k}=\beta_{k} \rho I
$$

and we note that $\Sigma_{k} \rho_{k} I_{k}=\rho I$. Together with the known values of $\rho_{k}$ and $I$, knowledge of $\alpha_{k}$ and $\beta_{k}$ is therefore sufficient to determine $\widetilde{\rho}_{k}$ and $I_{k}$, which are the natural independent thermodynamic variables for material $k$ in isolation. The state relations for pure material $k$ are also presumed known, so $\widetilde{\rho}_{k}$ and $I_{k}$ then determine the remaining thermodynamic properties of material $k$, such as its pressure $\widetilde{p}_{k}$, temperature $T_{k}$, and so on.

Two problems now remain: (a) how to determine $\alpha_{k}$ and $\beta_{k}$, thereby determining $\widetilde{p}_{k}, T_{k}$, etc., and (b) having done so, how to compute the thermodynamic properties of the mixture, such as its pressure $p$ and temperature $T$, in terms of the thermodynamic properties of the individual materials $k$ of which it is composed. In order to determine the $2 N$ -2 variables $\alpha_{k}$ and $\beta_{k}$, it is necessary to impose $2 N-2$ conditions. The most common, natural, and intuitive choice for these conditions is to require that the subvolumes are in pressure and temperature equilibrium with each other. Requiring all the subvolume pressures $\widetilde{p}_{k}$ to be equal imposes $N-1$ conditions, and requiring all their temperatures $T_{k}$ to be equal imposes another $N-1$, so this provides the $2 N$ -2 conditions needed to determine the values of $\alpha_{k}$ and $\beta_{k}$, thereby solving problem (a). Moreover, it is natural to identify the resulting common value of the $\widetilde{p}_{k}$ with the pressure $p$ of the mixture, and that of the $T_{k}$ with the temperature $T$ of the mixture, thereby solving problem (b) as well. This pro- 
cedure seems intuitively reasonable, but of course it represents an uncontrolled approximation in general. (The true state relations for a dense gas mixture depend on the form of the interaction potential between atoms and ions of different materials, and this information does not enter into the state relations of the pure materials.) However, it is easy to show, and appears to be well known, that this procedure is actually exact for a mixture of neutral (nonionized) ideal gases, and produces precisely the correct thermal and caloric state equations for such a mixture (see the Appendix). This encourages the hope that the same procedure will also provide a reasonable approximation to the state relations of nonideal mixtures.

Unfortunately, the above procedure does not produce the correct state relations for a mixture of partially ionized ideal gases, as will be shown in the next section. The physical reason for this is simply that the electrons produced by the ionization of one material tend to suppress the ionization of the others, and vice versa, and this effect is not accounted for in the procedure described above. The purpose of this paper is to show that this problem may be removed simply by equating the free electron densities of the subvolumes instead of their pressures. When this is done, the procedure thus modified reproduces the correct state relations for a mixture of partially ionized ideal gases. Moverover, this remedy is remarkably general; it applies to a mixture of an arbitrary number of materials, even in the presence of multiple ionization and partially degenerate and/or relativistic electrons. It also remains valid when used in conjunction with a common approximation for pressure ionization, as well as in two-temperature plasmas. Of course, this procedure, like pressure equilibration, becomes an uncontrolled approximation for dense mixtures, in which the atoms and ions of different materials interact with each other. However, the fact that it is at least exact for ideal mixtures, in contrast to pressure equilibration, suggests that it is likely to provide a better approximation in nonideal (dense) partially ionized gas mixtures.

In ideal systems, equilibrating subvolume temperatures and free electron number densities is equivalent to equilibrating temperatures and electron chemical potentials. This equivalence no longer obtains in dense systems, where one would intuitively expect that it is the chemical potentials that should be equilibrated, since differences between them are the general thermodynamic driving forces associated with mass exchange between subsystems, and in the present context the artificial partitions separating the subvolumes may be thought of as semipermeable membranes through which free electrons may pass but heavy particles may not. The equilibration of electron number density, or chemical potential, instead of pressure has previously been proposed on intuitive grounds, but we are unaware of a previous published justification for this procedure.

The present discussion is organized as follows. In Sec. II we consider mixtures of classical partially ionized ideal gases, and show that equilibrating subvolume pressures and temperatures leads to incorrect mixture state relations, while equating subvolume temperatures and free electron densities reproduces the correct state relations. In Sec. III these results are generalized to the case of partially degenerate and/or relativistic electrons, and to a common approximation for pressure ionization. The case of two-temperature plasmas, in which the electron temperature $T_{e}$ differs from the heavy particle temperature, is discussed in Sec. IV. Section V contains a few concluding remarks.

\section{CLASSICAL IDEAL GASES}

\section{A. The true mixture}

We consider a classical ideal gas mixture of $N$ different types of atoms denoted by the symbols $X_{k}(k=1,2, \ldots, N)$, the various ionization states of which are denoted by $X_{k}^{n}(n$ $\left.=0,1,2, \ldots, N_{k}\right)$, so that $X_{k}^{0}$ refers to neutral atoms of $X_{k}$ while $X_{k}^{n}$ for $n \geqslant 1$ refers to $n$-tuply ionized $X_{k}$, i.e., $X_{k}^{1}$ $=X_{k}^{+}, X_{k}^{2}=X_{k}^{++}$, etc. The free electrons will simply be denoted by the symbol $e$, which will also be used as a sub- or superscript as convenient. The mass of a single particle of $X_{k}^{n}$ is denoted by $m_{k}^{n}$, and clearly $m_{k}^{n}=m_{k}^{0}-n m_{e}$, where $m_{k}^{0}$ is the mass of a single neutral atom of $X_{k}^{0}$ and $m_{e}$ is the mass of a single electron. The partial mass density of $X_{k}$ in the mixture is denoted by $\rho_{k}$, which is presumed to be a known given quantity and is of course unchanged by ionization. Thus $\rho_{k}$ includes the mass of all electrons originally present in $X_{k}$, regardless of whether they remain bound or have become free due to ionization. The partial number density of $X_{k}$ in the absence of ionization is then $n_{k}=\rho_{k} / m_{k}^{0}$, which is therefore also a known quantity. The partial mass density of $X_{k}^{n}$ in the partially ionized mixture is denoted by $\rho_{k}^{n}$ $=m_{k}^{n} n_{k}^{n}$, where $n_{k}^{n}$ is the corresponding partial number density. Similarly, the partial mass density of free electrons in the mixture, produced by ionization of all materials present, is $\rho_{e}=m_{e} n_{e}$, where $n_{e}$ is the partial number density of free electrons in the mixture. Since the particle masses are known, the partial mass and number densities carry equivalent information, but it will usually be more convenient to work in terms of the latter.

Now ionization produces free electrons but does not change the number of heavy particles present, so the total number density of heavy particles of each material is unchanged by ionization and remains $n_{k}$ even in a partially ionized mixture. The number densities $n_{k}^{n}$ are therefore constrained by the $N$ equations

$$
\sum_{n} n_{k}^{n}=n_{k} .
$$

Note that $n_{k}$ accordingly does not include the number density of free electrons produced by ionization of $X_{k}$, whereas in contrast, $\rho_{k}$ does include the mass of those electrons as already discussed. In addition, conservation of electrons implies the condition

$$
\sum_{k n} n n_{k}^{n}=n_{e} .
$$

The specific internal energy (energy per unit mass) $I$ of the mixture is also presumed known, and is related to the specific internal energies of the individual species by 


$$
\sum_{k n} m_{k}^{n} n_{k}^{n} I_{k}^{n}(T)+m_{e} n_{e} I_{e}(T)=\rho I,
$$

where $T$ is the temperature, and $I_{k}^{n}(T)$ and $I_{e}(T)$ are the caloric equations of state for the heavy particles and electrons, respectively, including their heats of formation, or in the present context ionization energies. We assume that the mixture is in ionization equilibrium, which implies that the number densities are further constrained by $N_{s}$ Saha equations of the form ${ }^{1}$

$$
\frac{n_{k}^{n+1} n_{e}}{n_{k}^{n}}=K_{k}^{n}(T) \quad\left(1 \leqslant k \leqslant N ; 0 \leqslant n \leqslant N_{k}-1\right),
$$

where $N_{s}=\Sigma_{k} N_{k}$ and the quantities $K_{k}^{n}(T)$ are equilibrium constants ${ }^{1}$ which are known functions of $T$.

Equations (3)-(6) constitute $N+1+1+N_{s}=N_{s}+N+2$ equations in the unknown variables $n_{k}^{n}, n_{e}$, and $T$. There are $N_{h}$ variables $n_{k}^{n}$, where $N_{h}=\Sigma_{k}\left(N_{k}+1\right)=N_{s}+N$, so there are $N_{h}+2=N_{s}+N+2$ unknowns. The system of Eqs. (3)(6) is therefore closed and the solution is determinate. Once these equations have been solved and $T$ is known, the pressure of the mixture is then given by the thermal equation of state for an ideal gas mixture, namely

$$
p=\sum_{k n} n_{k}^{n} k_{\mathrm{B}} T+n_{e} k_{\mathrm{B}} T=\sum_{k} n_{k} k_{\mathrm{B}} T+n_{e} k_{\mathrm{B}} T,
$$

where $k_{\mathrm{B}}$ is Boltzmann's constant. Equations (3)-(7) therefore implicitly determine both the pressure $p$ and temperature $T$ of the mixture as a function of the variables $\left(\rho_{k}, I\right)$.

\section{B. The partitioned mixture}

We now suppose that the mixture is artificially partitioned into subvolumes as described in the Introduction. According to Eqs. (1) and (2), the density of material $X_{k}$ within its subvolume is then $\widetilde{\rho}_{k}=\rho_{k} / \alpha_{k}$, while its specific internal energy is $I_{k}=\beta_{k} \rho I / \rho_{k}$. In the absence of ionization, the number density of material $k$ within its subvolume would then be $\widetilde{n}_{k}=\widetilde{\rho}_{k} / m_{k}^{0}=\rho_{k} /\left(\alpha_{k} m_{k}^{0}\right)=n_{k} / \alpha_{k}$. The number density of $X_{k}^{n}$ within material $k$ is denoted by $\widetilde{n}_{k}^{n}$, and the number density of free electrons within material $k$ is denoted by $\widetilde{n}_{k}^{e}$. The total number density of heavy particles of material $k$ is again unchanged by ionization, so the number densities $\widetilde{n}_{k}^{n}$ for each material $k$ are constrained by

$$
\sum_{n} \widetilde{n}_{k}^{n}=\widetilde{n}_{k}=n_{k} / \alpha_{k},
$$

while conservation of electrons for material $k$ implies the additional condition

$$
\sum_{n} n \widetilde{n}_{k}^{n}=\widetilde{n}_{k}^{e}
$$

The specific internal energy of material $k$ within its subvolume is related to the specific internal energies of its individual species by

$$
\begin{aligned}
\sum_{n} m_{k}^{n} \widetilde{n}_{k}^{n} I_{k}^{n}\left(T_{k}\right)+m_{e} \widetilde{n}_{k}^{e} I_{e}\left(T_{k}\right) & =\widetilde{\rho}_{k} I_{k}=\widetilde{\rho}_{k} \beta_{k} \rho I / \rho_{k} \\
& =\beta_{k} \rho I / \alpha_{k} .
\end{aligned}
$$

The assumption of ionization equilibrium further implies that the number densities within material $k$ are constrained by the $N_{k}$ Saha equations

$$
\frac{\widetilde{n}_{k}^{n+1} \widetilde{n}_{k}^{e}}{\widetilde{n}_{k}^{n}}=K_{k}^{n}\left(T_{k}\right) \quad\left(0 \leqslant n \leqslant N_{k}-1\right) .
$$

The number densities $\widetilde{n}_{k}^{n}$ and $\widetilde{n}_{k}^{e}$ within material $k$ are particle numbers per unit volume of material $k$. In order to facilitate comparison with the true mixture relations, it is convenient to eliminate these number densities in favor of the corresponding partial number densities (particle numbers per unit total volume) $n_{k}^{n}=\alpha_{k} \widetilde{n}_{k}^{n}$ and $n_{k}^{e}=\alpha_{k} \widetilde{n}_{k}^{e}$. Equations (8)-(11) then become

$$
\begin{aligned}
& \sum_{n} n_{k}^{n}=n_{k}, \\
& \sum_{n} n n_{k}^{n}=n_{k}^{e}, \\
& \sum_{n} m_{k}^{n} n_{k}^{n} I_{k}^{n}\left(T_{k}\right)+m_{e} n_{k}^{e} I_{e}\left(T_{k}\right)=\beta_{k} \rho I, \\
& \frac{n_{k}^{n+1} n_{k}^{e}}{n_{k}^{n}}=\alpha_{k} K_{k}^{n}\left(T_{k}\right) \quad\left(0 \leqslant n \leqslant N_{k}-1\right) .
\end{aligned}
$$

If $\alpha_{k}$ and $\beta_{k}$ were known, Eqs. (12)-(15) would constitute $N_{k}+3$ equations in the $N_{k}+3$ unknowns $n_{k}^{n}, n_{k}^{e}$, and $T_{k}$ for each material $k$, and the pressure $\widetilde{p}_{k}$ within material $k$ would then be given by $\widetilde{p}_{k}=\Sigma_{n} \widetilde{n}_{k}^{n} k_{\mathrm{B}} T_{k}+\widetilde{n}_{k}^{e} k_{\mathrm{B}} T_{k}$, or

$$
\alpha_{k} \widetilde{p}_{k}=\sum_{n} n_{k}^{n} k_{\mathrm{B}} T_{k}+n_{k}^{e} k_{\mathrm{B}} T_{k}=\left(n_{k}+n_{k}^{e}\right) k_{\mathrm{B}} T_{k} .
$$

In order to determine the variables $\alpha_{k}$ and $\beta_{k}$ for all $k$, we must impose an additional $2 N-2$ conditions. Since the quantities $K_{k}^{n}(T)$ and $I_{k}^{n}(T)$ are in general nonlinear functions of $T$, if we are to have any hope of reproducing the true solution for the mixture it is obvious that we must require temperature equilibrium between the subvolumes, i.e., $T_{k}$ $=T_{1} \equiv T$ for $k=2, \ldots, N$. This provides $N-1$ conditions which we may regard as determining the variables $\beta_{k}$. Equations (14)-(16) then become

$$
\begin{aligned}
& \sum_{n} m_{k}^{n} n_{k}^{n} I_{k}^{n}(T)+m_{e} n_{k}^{e} I_{e}(T)=\beta_{k} \rho I, \\
& \frac{n_{k}^{n+1} n_{k}^{e}}{n_{k}^{n}}=\alpha_{k} K_{k}^{n}(T) \quad\left(0 \leqslant n \leqslant N_{k}-1\right), \\
& \alpha_{k} \widetilde{p}_{k}=\left(n_{k}+n_{k}^{e}\right) k_{\mathrm{B}} T .
\end{aligned}
$$

The system of equations to be solved now consists of Eqs. (12), (13), and (17)-(19) for all $k$. The total number of these equations is $N+N+N+N_{s}+N=N_{s}+4 N$, and if the $\alpha_{k}$ were known the remaining unknown quantities would be $n_{k}^{n}$, 
$n_{k}^{e}, T, \beta_{k}$, and $\tilde{p}_{k}$, the total number of which is $N_{h}+N$ $+1+(N-1)+N=N_{h}+3 N=N_{s}+4 N$. The system is therefore determinate for given $\alpha_{k}$.

Let us now see how close we are to achieving consistency with the corresponding relations for the true mixture, namely Eqs. (3)-(7). Equation (12) is already of the same form as Eq. (3), while summing Eq. (13) over $k$ yields

$$
\sum_{k n} n n_{k}^{n}=n_{e}
$$

where $n_{e}=\sum_{k} n_{k}^{e}$ is the total number density of free electrons, i.e., the free electrons produced by ionization of all materials per unit total volume. Equation (20) is seen to be of the same form as Eq. (4). Summing Eq. (17) over $k$ reproduces Eq. (5), and summing Eq. (19) over $k$ reproduces Eq. (7) provided that we let $p=\Sigma_{k} \alpha_{k} \widetilde{p}_{k}$. Thus we already have consistency with Eqs. (3) $-(5)$ and (7) even though the $\alpha_{k}$ still remain arbitrary, and the only remaining question is whether we can determine them in such a way that the partitioned Saha equations (18) are consistent with the true mixture Saha equations (6). Of course this does not require that the $\alpha_{k}$ be specified explicitly in closed form; they can also be implicitly determined by imposing an additional $N-1$ independent conditions on the unknown variables $n_{k}^{n}, n_{k}^{e}, T, \alpha_{k}, \beta_{k}$, and/or $\widetilde{p}_{k}$. Comparison of Eqs. (6) and (18) shows that these equations would indeed be consistent if these additional $N$ -1 conditions can be shown to imply the relations $\alpha_{k}$ $=n_{k}^{e} / n_{e}$, or

$$
n_{k}^{e}=\alpha_{k} n_{e} \text {. }
$$

Let us see if equating the subvolume pressures produces the desired effect, since we know that this works for mixtures of neutral nonionized ideal gases (see the Appendix). Thus we set $\widetilde{p}_{k}=\widetilde{p}_{1} \equiv p$ for $k=2, \ldots, N$, whereupon Eq. (19) becomes $\alpha_{k} p=\left(n_{k}+n_{k}^{e}\right) k_{\mathrm{B}} T$, so that

$$
\alpha_{k}=\frac{\left(n_{k}+n_{k}^{e}\right) k_{\mathrm{B}} T}{\left(\sum_{j} n_{j}+n_{e}\right) k_{\mathrm{B}} T}=\frac{n_{k}+n_{k}^{e}}{\sum_{j} n_{j}+n_{e}} .
$$

Solving for $n_{k}^{e}$, we find

$$
n_{k}^{e}=\alpha_{k} n_{e}+\alpha_{k} \sum_{j} n_{j}-n_{k}
$$

which differs from Eq. (21). Equating the subvolume pressures therefore results in an inconsistency between the partitioned Saha equations (15) and the true mixture Saha equations (6), and this of course destroys the desired consistency between the partitioned and true mixture equations as a whole.

We observe that Eqs. (21) and (23) would no longer differ if it could somehow be shown that $\alpha_{k}=n_{k} / \Sigma_{j} n_{j}$ as well. We further observe, however, that this cannot in general be true, since the $n_{k}$ are known given quantities independent of $T$, whereas it is clear that $n_{k}^{e} / n_{e}$ will in general depend strongly on $T$ via the equilibrium constants $K_{k}^{n}$.

Fortunately, the inconsistency is easily remedied. Only $N-1$ of the relations (21) required to achieve the desired consistency are independent, since their sum over $k$ reduces to an identity. The relations (21) themselves can therefore be imposed as the additional conditions required to determine the $\alpha_{k}$, and when this is done Eqs. (6) and (15) immediately and automatically become consistent. Moreover, Eq. (21) is equivalent to $\widetilde{n}_{k}^{e}=n_{e}$, which simply states that the free electron densities of all the different materials within their respective subvolumes are equal. Equating these free electron densities instead of pressures thereby produces full consistency of the equations for the partitioned mixture with those of the true mixture. Of course, the resulting subvolume pressures $\widetilde{p}_{k}$ will then no longer be equal, and the correct mixture pressure is then simply given by $p=\Sigma_{k} \alpha_{k} \widetilde{p}_{k}$ as discussed above. Note that the quantities $p_{k} \equiv \alpha_{k} \widetilde{p}_{k}$ play the role of partial pressures, since their sum is the total pressure $p$ of the mixture.

\section{DEGENERATE OR RELATIVISTIC ELECTRONS AND PRESSURE IONIZATION}

The development of the preceding section was restricted to classical (Maxwell-Boltzmann) statistics. In many applications, however, the electrons may be partially degenerate quantum-mechanically, or relativistic, or both, and the development requires modifications. In particular, the specific internal energy of the free electrons, and the ionization equilibrium constants in the Saha equations, no longer depend on $T$ alone but acquire a dependence on $n_{e}$ as well. ${ }^{1}$ Equation (5) for the true mixture is therefore replaced by

$$
\sum_{k n} m_{k}^{n} n_{k}^{n} I_{k}^{n}(T)+m_{e} n_{e} I_{e}\left(T, n_{e}\right)=\rho I,
$$

while Eq. (17) for the partitioned mixture is replaced by

$$
\sum_{n} m_{k}^{n} n_{k}^{n} I_{k}^{n}(T)+m_{e} n_{k}^{e} I_{e}\left(T, n_{k}^{e} / \alpha_{k}\right)=\beta_{k} \rho I,
$$

where the precise functional form of $I_{e}\left(T, n_{e}\right)$ is immaterial for present purposes.

Similarly, Eq. (6) for the true mixture is replaced by

$$
\frac{n_{k}^{n+1} n_{e}}{n_{k}^{n}}=K_{k}^{n}\left(T, n_{e}\right),
$$

while Eq. (18) for the partitioned mixture is replaced by

$$
\frac{n_{k}^{n+1} n_{k}^{e}}{n_{k}^{n}}=\alpha_{k} K_{k}^{n}\left(T, n_{k}^{e} / \alpha_{k}\right),
$$

where the precise functional form of $K_{k}^{n}\left(T, n_{e}\right)$ is also immaterial for present purposes. The final such modification is that Eq. (7) for the true mixture is replaced by

$$
p=\sum_{k} n_{k} k_{\mathrm{B}} T+p_{e}\left(n_{e}, T\right)
$$

while Eq. (19) for the partitioned mixture is replaced by

$$
\alpha_{k} \widetilde{p}_{k}=n_{k} k_{\mathrm{B}} T+\alpha_{k} p_{e}\left(n_{k}^{e} / \alpha_{k}, T\right)
$$

and the precise functional form of the electron pressure $p_{e}\left(n_{e}, T\right)$ is again immaterial.

Let us now see if the relations (21) still suffice to ensure the desired consistency between the true and partitioned mixture relations. Combining Eqs. (21) and (25) and summing 
over $k$, we obtain precisely Eq. (24), while combining Eqs. (21) and (27) reproduces Eq. (26). Finally, combining Eqs. (21) and (29) and summing over $k$, we obtain precisely Eq. (28) provided that we let $p=\Sigma_{k} \alpha_{k} \widetilde{p}_{k}$ as before. The relations (21) therefore still suffice to ensure the desired consistency, and hence can still be employed as the additional conditions required to determine the $\alpha_{k}$ for this purpose.

A similar situation obtains with regard to a common approximation used to represent pressure ionization effects, ${ }^{2}$ wherein those effects are modeled by introducing a further approximate dependence on the free electron density into the ionization equilibrium constants $K_{k}^{n}$. The functional form of this dependence is again immaterial for present purposes; whatever it is, it may simply be incorporated into the functions $K_{k}^{n}\left(T, n_{e}\right)$, so that Eqs. (26) and (27) continue to apply and the consistency conditions (21) again remain unchanged.

\section{TWO-TEMPERATURE PLASMAS}

In many situations of interest, the temperature $T_{e}$ of the free electrons differs from the temperature $T$ of the heavy particles, and the development then requires still further modifications. In this case, $T_{e}$ is determined by a separate evolution equation for the specific internal energy $I_{e}$ of the free electrons, which therefore becomes an additional known quantity in the description. In the true mixture, we then have

$$
I_{e}\left(T_{e}, n_{e}\right)=I_{e}^{0},
$$

where $I_{e}^{0}$ is the known given value of $I_{e}$ as determined by the electron energy equation. Equation (24) for the total energy density in the true mixture, including that of the free electrons, then becomes

$$
\begin{aligned}
\sum_{k n} & m_{k}^{n} n_{k}^{n} I_{k}^{n}(T)+m_{e} n_{e} I_{e}\left(T_{e}, n_{e}\right) \\
& =\sum_{k n} m_{k}^{n} n_{k}^{n} I_{k}^{n}(T)+m_{e} n_{e} I_{e}^{0} \\
& =\rho I .
\end{aligned}
$$

In the artificially partitioned mixture, each material now has its own electron temperature $T_{k}^{e}$, and it becomes necessary to partition the electron energy density as well as the total internal energy density. For this purpose we introduce electron energy partitioning parameters $\beta_{k}^{e}$ analogous to the $\beta_{k}$, so that

$$
n_{k}^{e} I_{e}\left(T_{k}^{e}, n_{k}^{e} / \alpha_{k}\right)=\beta_{k}^{e} n_{e} I_{e}^{0},
$$

while Eq. (25) for the partitioned mixture now becomes

$$
\begin{aligned}
\sum_{n} m_{k}^{n} n_{k}^{n} I_{k}^{n}(T)+m_{e} n_{k}^{e} I_{e}\left(T_{k}^{e}, n_{k}^{e} / \alpha_{k}\right) \\
=\sum_{n} m_{k}^{n} n_{k}^{n} I_{k}^{n}(T)+m_{e} n_{e} \beta_{k}^{e} I_{e}^{0} \\
=\beta_{k} \rho I .
\end{aligned}
$$

The Saha equations in a two-temperature plasma have been controversial, ${ }^{3-15}$ and we shall not attempt to resolve those controversies here. We note, however, that most of the competing variants in the literature have in common the feature that the ionization equilibrium constants $K_{k}^{n}$ become functions of $T_{e}$ as well as $T$ and $n_{e}$. The precise mathematical form of this additional functional dependence is immaterial for present purposes, so the present development applies equally well to any particular two-temperature Saha equation of this general form. The Saha equations (26) for the true mixture then become

$$
\frac{n_{k}^{n+1} n_{e}}{n_{k}^{n}}=K_{k}^{n}\left(T, T_{e}, n_{e}\right),
$$

while the corresponding equations (27) for the artificially partitioned mixture become

$$
\frac{n_{k}^{n+1} n_{k}^{e}}{n_{k}^{n}}=\alpha_{k} K_{k}^{n}\left(T, T_{k}^{e}, n_{k}^{e} / \alpha_{k}\right) .
$$

In a two-temperature plasma, Eq. (28) for the true mixture pressure is replaced by

$$
p=\sum_{k} n_{k} k_{\mathrm{B}} T+p_{e}\left(n_{e}, T_{e}\right)
$$

while Eq. (29) for the subvolume pressures in the partitioned mixture becomes

$$
\alpha_{k} \widetilde{p}_{k}=n_{k} k_{\mathrm{B}} T+\alpha_{k} p_{e}\left(n_{k}^{e} / \alpha_{k}, T_{k}^{e}\right) .
$$

Guided by the preceding development, we may anticipate that the $\beta_{k}^{e}$ should be determined by requiring the electron temperatures of all materials to be equal, i.e., $T_{k}^{e}=T_{1}^{e}$ $\equiv T_{e}$ for $k=2, \ldots, N$. Equations (32) and (33) then become

$$
\begin{aligned}
& n_{k}^{e} I_{e}\left(T_{e}, n_{k}^{e} / \alpha_{k}\right)=\beta_{k}^{e} n_{e} I_{e}^{0}, \\
& \sum_{n} m_{k}^{n} n_{k}^{n} I_{k}^{n}(T)+m_{e} n_{e} \beta_{k}^{e} I_{e}^{0}=\beta_{k} \rho I,
\end{aligned}
$$

while the Saha equations (35) become

$$
\frac{n_{k}^{n+1} n_{k}^{e}}{n_{k}^{n}}=\alpha_{k} K_{k}^{n}\left(T, T_{e}, n_{k}^{e} / \alpha_{k}\right)
$$

and Eq. (37) becomes

$$
\alpha_{k} \widetilde{p}_{k}=n_{k} k_{\mathrm{B}} T+\alpha_{k} p_{e}\left(n_{k}^{e} / \alpha_{k}, T_{e}\right) .
$$

We now check to see if the conditions (21) still suffice to ensure consistency between Eqs. (30) and (31) for the true mixture and Eqs. (38) and (39) for the partitioned mixture. Combining Eq. (21) with Eqs. (38) and (39), we obtain

$$
\alpha_{k} n_{e} I_{e}\left(T_{e}, n_{e}\right)=\beta_{k}^{e} n_{e} I_{e}^{0},
$$

while Eq. (39) remains unchanged. Summing Eqs. (42) and (39) over $k$, we obtain precisely Eqs. (30) and (31), so the desired consistency obtains for the energies.

The next step is to see if the conditions (21) also suffice to ensure consistency between Eq. (34) for the true mixture and Eq. (40) for the partitioned mixture. Combining Eqs. (21) and (40), we obtain precisely Eq. (34), thereby confirming the desired consistency for the Saha equations. Finally, combining Eq. (21) with Eq. (41) and summing over $k$, we obtain 


$$
\sum_{k} \alpha_{k} \widetilde{p}_{k}=\sum_{k} n_{k} k_{\mathrm{B}} T+p_{e}\left(n_{e}, T_{e}\right),
$$

which agrees precisely with Eq. (36) provided that we again let $p=\Sigma_{k} \alpha_{k} \widetilde{p}_{k}$. The conditions (21) therefore again produce full consistency between the relations for the true and partitioned mixtures.

\section{CONCLUSION}

We have shown that equating the temperatures and free electron densities (or equivalently chemical potentials) in the subvolumes of an artificially partitioned mixture of partially ionized ideal gases reproduces the correct thermal and caloric state equations of the true mixture, even when the electrons are partially degenerate and/or relativistic and/or their temperature differs from that of the heavy particles. It should be noted, however, that the entropy of the true mixture, and hence the other thermodynamic potentials (free energies) defined in terms of it, differs from the sum of the subvolume entropies by an entropy of mixing, but this difference can readily be evaluated and accounted for in ideal systems. The total entropy per unit volume of the true mixture is given by

$$
\sigma=\sum_{k n} n_{k}^{n}\left[s_{k n}^{0}(T)-k_{\mathrm{B}} \ln n_{k}^{n}\right]+\sigma_{e}\left(n_{e}, T_{e}\right),
$$

where $s_{k n}^{0}(T)$ is a function of $T$ alone, and $\sigma_{e}\left(n_{e}, T_{e}\right)$ is the entropy per unit volume of the free electrons, which again may be partially degenerate and/or relativistic. The entropy of material $k$ per unit volume of material $k$ within the artificially partitioned mixture is similarly given by

$$
\widetilde{\sigma}_{k}=\sum_{n} \widetilde{n}_{k}^{n}\left[s_{k n}^{0}(T)-k_{\mathrm{B}} \ln \widetilde{n}_{k}^{n}\right]+\sigma_{e}\left(\widetilde{n}_{k}^{e}, T_{e}\right) .
$$

The total entropy per unit total volume of the artificially partitioned mixture is therefore given by

$$
\begin{aligned}
\sigma^{\prime}=\sum_{k} \alpha_{k} \widetilde{\sigma}_{k}= & \sum_{k n} n_{k}^{n}\left[s_{k n}^{0}(T)-k_{\mathrm{B}} \ln \left(n_{k}^{n} / \alpha_{k}\right)\right] \\
& +\sum_{k} \alpha_{k} \sigma_{e}\left(\widetilde{n}_{k}^{e}, T_{e}\right) .
\end{aligned}
$$

As already shown, equating the electron number densities of the subvolumes implies that $\widetilde{n}_{k}^{e}=n_{e}$, so that Eq. (46) becomes

$$
\begin{aligned}
\sigma^{\prime} & =\sum_{k n} n_{k}^{n}\left[s_{k n}^{0}(T)-k_{\mathrm{B}} \ln n_{k}^{n}+k_{\mathrm{B}} \ln \alpha_{k}\right]+\sigma_{e}\left(n_{e}, T_{e}\right) \\
& =\sigma+k_{\mathrm{B}} \sum_{k} n_{k} \ln \alpha_{k},
\end{aligned}
$$

in which the last term is the aforementioned entropy of mixing, which is seen to be simple in form and easily evaluated. Equation (47) relates the entropy per unit volume of the true mixture to that of the artificially partitioned mixture, thereby allowing the former to be calculated from the latter.

Since the thermal and caloric state equations resulting from the present procedure are exact for ideal systems, they are ipso facto thermodynamically consistent. In nonideal (dense) systems, where the present procedure becomes approximate, it intuitively seems preferable to equilibrate the electron chemical potentials rather than the number densities, and since the resulting state relations are no longer exact, thermodynamic consistency is no longer so obvious. However, it may be argued that thermodynamic consistency should be preserved by virtue of the fact that the artificially partitioned mixture, although admittedly different from the true mixture, may nevertheless be regarded as a real albeit idealized physical system in which the partitions separating the subvolumes are semipermeable membranes with pores so small that only the free electrons, but not the heavy particles, may pass through them. As is well known, the chemical potential of any species that can pass through such a membrane has the same value on both sides, so equating the electron chemical potentials is indeed the physically correct condition to impose in this situation. This procedure should therefore produce the physically correct state relations for the partitioned system, and if so those state relations should ipso facto be thermodynamically consistent as well, even though they are only an approximation to those of the true nonideal mixture.

\section{ACKNOWLEDGMENTS}

I am grateful to John Castor, C. H. Chang, Larry Cloutman, Peter Eggleton, Tom McAbee, David Young, and George Zimmerman for helpful discussions and comments on the manuscript.

This work was performed under the auspices of the U.S. Department of Energy by the University of California Lawrence Livermore National Laboratory under Contract No. W-7405-Eng-48.

\section{APPENDIX: NEUTRAL GAS MIXTURES}

Here we wish to show that equating the subvolume pressures and temperatures reproduces the correct state relations for a mixture of neutral (nonionized) ideal gases. The thermal and caloric equations of state for such a mixture are given by

$$
\begin{aligned}
& p=\sum_{k} n_{k} k_{\mathrm{B}} T, \\
& \rho I=\sum_{k} \rho_{k} I_{k}(T),
\end{aligned}
$$

where $k_{\mathrm{B}}$ is Boltzmann's constant, $n_{k}=\rho_{k} / m_{k}$ is the number density of material $k, m_{k}$ is its atomic mass, and $I_{k}(T)$ is its specific internal energy, which is presumed known as a function of $T$. Equation (A2) implicitly determines the mixture temperature $T$ as a function of the known independent variables $\rho_{k}$ and $I$. Substitution into Eq. (A1) then yields the mixture pressure $p$ as a function of the same variables.

Now suppose the mixture is artificially partitioned into subvolumes as described in the Introduction. According to Eqs. (1) and (2), the density of material $k$ within its subvolume is then $\widetilde{\rho}_{k}=\rho_{k} / \alpha_{k}$, while its specific internal energy is $I_{k}=\beta_{k} \rho I / \rho_{k}$. Thus the number density of material $k$ within 
its subvolume is $\tilde{n}_{k}=\tilde{\rho}_{k} / m_{k}=n_{k} / \alpha_{k}$, and the pressure and temperature of material $k$ within its subvolume are therefore given by

$$
\begin{aligned}
& \tilde{p}_{k}=\frac{n_{k} k_{\mathrm{B}} T_{k}}{\alpha_{k}}, \\
& \rho_{k} I_{k}\left(T_{k}\right)=\beta_{k} \rho I .
\end{aligned}
$$

If the $\alpha_{k}$ and $\beta_{k}$ were known, Eqs. (A3) and (A4) would constitute $2 N$ equations in the $2 N$ unknowns $\widetilde{p}_{k}$ and $T_{k}$. In order to determine $\alpha_{k}$ and $\beta_{k}$ as well, we require an additional $2 N-2$ equations, which we obtain by requiring the subvolumes to be in pressure and temperature equilibrium, i.e., $\widetilde{p}_{k}=\widetilde{p}_{1} \equiv p$ and $T_{k}=T_{1} \equiv T$ for $k=2, \ldots, N$. Equations (A3) and (A4) then become

$$
\begin{aligned}
& \alpha_{k} p=n_{k} k_{\mathrm{B}} T, \\
& \beta_{k} \rho I=\rho_{k} I_{k}(T) .
\end{aligned}
$$

Summing these equations over $k$, we obtain precisely Eqs. (A1) and (A2). The variables $p$ and $T$ computed by artificially partitioning the system and equilibrating subvolume temperatures and pressures are therefore identical to the correct pressure and temperature of the true unpartitioned mixture.

It is noteworthy that the pressure equilibration, while intuitively appealing, is not actually necessary. If we simply set $T_{k}=T_{1} \equiv T$ to determine the $\beta_{k}$ but leave the volume fractions $\alpha_{k}$ arbitrary, Eq. (A6) is unchanged while Eq. (A5) is replaced by

$$
\alpha_{k} \widetilde{p}_{k}=n_{k} k_{\mathrm{B}} T \text {. }
$$

Summing Eq. (A6) over $k$ then yields Eq. (A2) as before, while summing Eq. (A7) over $k$ yields Eq. (A1) with $p$ replaced by $\Sigma_{k} \alpha_{k} \widetilde{p}_{k}$. The quantity $p=\Sigma_{k} \alpha_{k} \widetilde{p}_{k}$ in the partitioned system is therefore the same as the correct pressure of the mixture, regardless of the values of the $\alpha_{k}$. Thus, even when the $\alpha_{k}$ are chosen arbitrarily and the subvolume pressures $\widetilde{p}_{k}$ are unequal, the correct pressure of the mixture may be obtained simply by computing the volume-weighted average of the subvolume pressures.

${ }^{1}$ J. P. Cox and R. T. Giuli, Principles of Stellar Structure (Gordon and Breach, New York, 1968), Vol. 1.

${ }^{2}$ P. P. Eggleton, J. Faulkner, and B. P. Flannery, Astron. Astrophys. 23, 325 (1973).

${ }^{3}$ M. Mitchner and C. H. Kruger, Jr., Partially Ionized Gases (WileyInterscience, New York, 1973).

${ }^{4}$ I. Prigogine, Bull. Cl. Sci., Acad. R. Belg. 26, 53 (1940).

${ }^{5}$ A. V. Potapov, High Temp. 4, 48 (1966).

${ }^{6}$ D. Kannappan and T. K. Bose, Phys. Fluids 20, 1668 (1977).

${ }^{7}$ M. L. Mittal and G. P. Gowda, J. Appl. Phys. 59, 1042 (1986).

${ }^{8}$ A. Morro and M. Romeo, J. Plasma Phys. 39, 41 (1988).

${ }^{9}$ M. C. M. van de Sanden, P. P. J. M. Schram, A. G. Peeters, J. A. M. van der Mullen, and G. M. W. Kroesen, Phys. Rev. A 40, 5273 (1989).

${ }^{10}$ V. Bakshi, Phys. Rev. A 42, 2460 (1990).

${ }^{11}$ M. C. M. van de Sanden, P. P. J. M. Schram, J. A. M. van der Mullen, and D. C. Schram, Phys. Rev. A 42, 2461 (1990).

${ }^{12}$ M. C. M. van de Sanden and P. P. J. M. Schram, Phys. Rev. A 44, 5150 (1991).

${ }^{13}$ J. A. M. van der Mullen, D. A. Benoy, F. H. A. G. Fey, B. van der Sijde, and J. Vlček, Phys. Rev. E 50, 3925 (1994).

${ }^{14}$ D. Giordano, Phys. Rev. E 58, 3098 (1998).

${ }^{15}$ D. Giordano and M. Capitelli, Phys. Rev. E 65, 016401 (2001). 